\title{
A shot in the dark...the nth shot!
}

\author{
Michele Di Mauro, MD, PhD, ${ }^{\mathrm{a}}$ Massimiliano Foschi, MD, ${ }^{\mathrm{b}}$ Fabrizio Tancredi, MD, ${ }^{\mathrm{b}}$ Sabina Gallina, MD, ${ }^{\mathrm{b}}$ \\ and Antonio M. Calafiore, $\mathrm{MD}^{\mathrm{c}}$
}

\footnotetext{
From the ${ }^{\mathrm{a} C a r d i o l o g y}$ and Cardiac Surgery, API Madonna del Ponte Institute, Lanciano; ${ }^{\mathrm{b}}$ Cardiovascular Disease Department, SS Annunziata Hospital and University of Chieti, Chieti; and ${ }^{\mathrm{c}}$ Cardiac Surgery, Pope John Paul II Foundation, Campobasso, Italy.

Disclosures: Authors have nothing to disclose with regard to commercial support.

Received for publication Oct 2, 2017; accepted for publication Oct 6, 2017; available ahead of print Nov 2, 2017

Address for reprints: Michele Di Mauro, MD, PhD, Cardiology and Cardiac Surgery, API Madonna del Ponte

Institute, Viale Cappuccini 50, Lanciano (CH) 66034, Italy (E-mail: mdimauro1973@gmail.com).

J Thorac Cardiovasc Surg 2018;155:1039-40

$0022-5223 / \$ 36.00$

Copyright (C) 2017 by The American Association for Thoracic Surgery

https://doi.org/10.1016/j.jtcvs.2017.10.026
}

Giving a rapid glance to the major guidelines on the management of heart valve diseases, we were unable to find any mention for tricuspid regurgitation (TR) in the 2017 focused update American Heart Association (AHA)/American College of Cardiology (ACC) Guidelines ${ }^{1}$ and some mild evidence-based suggestions in the other guidelines. ${ }^{2,3}$ In the 2014 AHA/ACC guidelines, "Tricuspid valve repair can be beneficial for patients with mild, moderate, or greater functional TR (stage B, early dilatation, moderate leaflet tethering) at the time of left-sided valve surgery with either 1) tricuspid annular dilation or 2) prior evidence of right $\mathrm{HF}$ (Level of Evidence LOE: B). Tricuspid valve repair may be considered for patients with moderate functional TR (stage B) and pulmonary artery hypertension at the time of leftsided valve surgery." To summarize the message, we "should" treat less than severe TR in the presence of tricuspid annular dilatation, and we "may" treat it in the presence of pulmonary hypertension. In the 2017 European Society of Cardiology/European Association for CardioThoracic Surgery guidelines, ${ }^{3}$ the suggestions are similar. We "should" treat less than severe TR in the presence of tricuspid annular dilatation, and we "may" treat it in the presence of recent right ventricular heart failure. In both cases, the level of evidence (LOE) was C. LOE B is based on a limited population evaluated and a single randomized trial or nonrandomized trial, and LOE C is based on the consensus of opinion of the experts or small studies, retrospective studies, and registries. Conversely, LOE $\mathrm{A}$ is achieved in the presence of multiple randomized clinical trials or meta-analyses for European Society of Cardiology and European Association for Cardio-Thoracic Surgery, more restrictive for the AHA and ACC, which assign LOE $\mathrm{A}$ in the presence of meta-analyses of high-quality randomized clinical trials. A meta-analysis ${ }^{4}$ including 2840 patients recently was performed demonstrating that the presence of more than moderate TR, TR progression, and TR grade were significantly lower in the TV repair group at a mean follow-up of 4.7 years. The same conclusions were reached in 2015 by Kara and colleagues. ${ }^{5}$

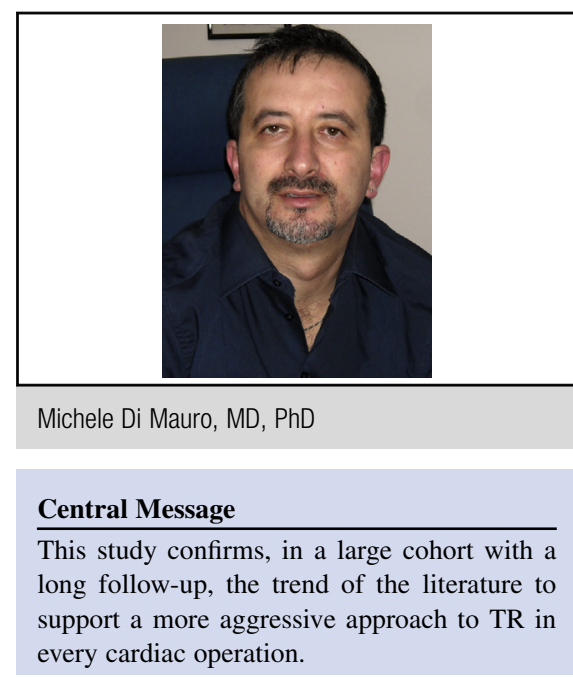

See Article page 1032 .

At this stage, so much evidence that TR may progress and impair prognosis, if not treated at the time of left-sided valve surgery, is not considered "so evident." In this "dark," the study by Kelly and colleagues ${ }^{6}$ represents the nth shot, another glare that unfortunately will not light up the scenario. However, this does not reduce the merit of the authors, who have done a very good job by focusing on the evaluation of what happens in the real world, with all that is included in dealing with the routine, which is not reproducible in a clinical trial. In this article, the impact of the severity of TR in more than 20,000 patients undergoing cardiac surgery, not just those undergoing left-sided valve surgery, is assessed, demonstrating that moderate TR has a significant influence on survival at 24 years. In the case of mild TR, statistical significance was not reached, but we are talking about a $P$ value of .06 . Moreover, they found that treating the tricuspid valve provided long-term survival. Finally, both right ventricle and left ventricle dysfunction were unfavorable predictors for late outcome.

Di Mauro and colleagues ${ }^{7}$ summarized the risk factors for worse late outcome and progression of TR over time. In patients undergoing left-sided valve surgery, moderate function TR has been identified, even if in smaller cohorts. In addition, older age, atrial fibrillation, huge left atrium, right ventricular dilatation and dysfunction, left ventricular dysfunction, preoperative New York Heart Association class, and pulmonary hypertension were identified as risk factors, but unfortunately none of them, except right ventricle dysfunction and pulmonary hypertension, are 
mentioned in the guidelines. Therefore, is it time for a trial to prove the necessity of treating moderate TR, whether it is primitive or secondary? The answer is inexorably yes, otherwise the LOE will remain low, despite having wellmade retrospective studies on a large population with a long follow-up, such as the present one. Thus, investigating as Inspector Clouseau on all research websites, we found 2 ongoing trials registered on clinicaltrial.gov. One is Evaluating the Benefit of Concurrent Tricuspid Valve Repair During Mitral Surgery from the Mayo Clinic (https://clinicaltrials.gov/ct2/show/NCT02675244). Unfortunately, among the inclusion criteria there is the following: "Undergoing MVS for degenerative MR with (a) Moderate TR as determined by transthoracic $2 \mathrm{D}$ echocardiography, or (b) tricuspid annular dimension $\geq 40 \mathrm{~mm}$ (index: $\geq 21 \mathrm{~mm} /$ $\mathrm{M}^{2} \mathrm{BSA}$ ) and trace/mild TR, determined by echocardiography." Moreover, patients with functional TRs are excluded. So, this trial focuses just on a small slice of the cake. Another ongoing trial is the Tricuspid Annuloplasty for Moderate Tricuspid Regurgitation Associated With Mitral Operation from the University of Maryland (https://clinicaltrials.gov/ct2/show/NCT01246947), for which the primary end point is the assessment of the degree of TR at 12 months after surgery in patients randomly assigned to isolated mitral valve surgery or mitral valve surgery plus tricuspid annuloplasty. This trial seems more able to clarify how to manage less than severe tricuspid surgery, but we should wait for longer follow-up.

Finally, let us point out 2 important concepts that inevitably arise from commenting not only on this article but also on the topic it introduces: the importance of anatomic more than functional evaluation of TR. As for functional mitral regurgitation, even in the case of functional TR, the grade is an index that may be fallacious, because it only mirrors the hemodynamic state of the moment but does not tell us anything about the fact that in hemodynamic distress a mild degree may become moderate and a moderate one may become severe; this condition affects the long-term prognosis of untreated patients. It is likely that, in the present study, some of the patients showing moderate TR had the anatomic stigma of severe TR, and the same is true for mild TR. We cannot exclusively trust the grade of $\mathrm{TR}$, as often happens in the routine. A more complex assessment of the annulus, tethering, size, and function of the right ventricle, pulmonary arterial pressure, atrium, and left ventricle should be performed. The other concept is how to treat TR to avoid a recurrence. Questions that we have already placed with the MR are as follows: Is a tricuspid annuloplasty enough? Is it so terrible to implant a prosthesis? Can we and should we develop new tricuspid repair techniques that are more effective and lasting over time?

Posterity will judge!

\section{References}

1. Nishimura RA, Otto CM, Bonow RO, Carabello BA, Erwin JP III, Fleisher LA, et al. 2017 AHA/ACC Focused Update of the 2014 AHA/ACC Guideline for the Management of Patients With Valvular Heart Disease: A Report of the American College of Cardiology/American Heart Association Task Force on Clinical Practice Guidelines. J Am Coll Cardiol. 2017;70:252-89.

2. Nishimura RA, Otto CM, Bonow RO, Carabello BA, Erwin JP III, Guyton RA, et al. American College of Cardiology; American College of Cardiology/American Heart Association; American Heart Association. 2014 AHA/ACC guideline for the management of patients with valvular heart disease: a report of the American College of Cardiology/American Heart Association Task Force on Practice Guidelines. J Thorac Cardiovasc Surg. 2014;148:e1-132.

3. Baumgartner H, Falk V, Bax JJ, De Bonis M, Hamm C, Holm PJ, et al. 2017 ESC/ EACTS Guidelines for the management of valvular heart disease: The Task Force for the Management of Valvular Heart Disease of the European Society of Cardiology (ESC) and the European Association for Cardio-Thoracic Surgery (EACTS). Eur Heart J. August 26, 2017 [Epub ahead of print].

4. Pagnesi M, Montalto C, Mangieri A, Agricola E, Puri R, Chiarito M, et al. Tricuspid annuloplasty versus a conservative approach in patients with functional tricuspid regurgitation undergoing left-sided heart valve surgery: a study-level meta-analysis. Int J Cardiol. 2017;240:138-44.

5. Kara I, Koksal C, Erkin A, Sacli H, Demirtas M, Percin B, et al. Outcomes of mild to moderate functional tricuspid regurgitation in patients undergoing mitral valve operations: a meta-analysis of 2,488 patients. Ann Thorac Surg. 2015;100: 2398-407.

6. Kelly BJ, Luxford JMH, Fanzca M, Goldberg Butler C, Huang CC, Wilusz K, et al. Severity of tricuspid regurgitation is associated with long term mortality. J Thorac Cardiovasc Surg. 2018;155:1032-8.e2.

7. Di Mauro M, Bezante GP, Di Baldassarre A, Clemente D, Cardinali A, Acitelli A, et al; Italian Study Group on Valvular Heart Disease Italian Society of Cardiology. Functional tricuspid regurgitation: an underestimated issue. Int J Cardiol. 2013; 168:707-15. 